\title{
Development of a multivariable prediction model to estimate the remaining lifespan of elderly patients with cerebral metastases from small-cell lung cancer
}

\author{
Dirk Rades $^{1}$, Trang Nguyen ${ }^{1}$, Stefan Janssen ${ }^{1}$, Steven E. Schild ${ }^{2}$ \\ ${ }^{1}$ Department of Radiation Oncology, University of Lübeck, Lübeck, Germany; ${ }^{2}$ Department of Radiation Oncology, Mayo Clinic, Scottsdale, \\ AZ, USA \\ Contributions: (I) Conception and design: All authors; (II) Administrative support: D Rades; (III) Provision of study materials or patients: D Rades, T \\ Nguyen, S Janssen; (IV) Collection and assembly of data: D Rades, T Nguyen, S Janssen; (V) Data analysis and interpretation: D Rades, SE Schild; (VI) \\ Manuscript writing: All authors; (VII) Final approval of manuscript: All authors. \\ Correspondence to: Prof. Dr. med. Dirk Rades. Chair of the Department of Radiation Oncology, University of Lübeck, Ratzeburger Allee 160, 23562 \\ Lübeck, Germany. Email: rades.dirk@gmx.net.
}

Background: Prognostic tools estimating survival of elderly patients with cerebral metastases from smallcell lung cancer (SCLC) improve treatment personalization. A specific tool for these patients was developed and compared to existing instruments.

Methods: One-hundred-and-forty elderly patients ( $\geq 65$ years) receiving whole-brain irradiation (WBI) for cerebral metastases from SCLC were retrospectively evaluated. WBI-program, age, gender, Karnofsky performance score, number of cerebral lesions, extracerebral metastases, and interval between SCLCdiagnosis and WBI were investigated. Characteristics significantly associated with survival in the multivariate analysis were used for the tool. Scoring points were calculated by dividing 6-month survival rates (\%) by 10 and added for patient scores. The tool was compared to existing diagnosis-specific instruments including updated diagnosis-specific graded prognostic assessment (DS-GPA), Rades-SCLC and WBRT-30-SCLC.

Results: In the multivariate analysis, KPS $(\mathrm{P}<0.001)$, number of cerebral lesions $(\mathrm{P}=0.013)$ and extracerebral metastases $(\mathrm{P}=0.049)$ were significantly associated with survival. Patient scores of $2(\mathrm{n}=37), 5$ $(\mathrm{n}=69), 8(\mathrm{n}=20)$ and $11(\mathrm{n}=14)$ points were obtained; 6-month survival rates were $0 \%, 9 \%, 50 \%$ and $79 \%$ $(\mathrm{P}<0.001)$. The positive predictive value $(\mathrm{PPV})$ of the worst group ( 2 points) to identify patients dying $\leq 6$ months was 100\%; PPVs of updated DS-GPA, Rades-SCLC and WBRT-30-SCLC were 94\%, 100\% and $94 \%$. PPV of the best group (11 points) to identify patients surviving $\geq 6$ months was $79 \%$; PPVs of updated DS-GPA, Rades-SCLC and WBRT-30-SCLC were 86\%, 79\% and 100\%.

Conclusions: The most precise instruments were the new tool and Rades-SCLC for identification of patients dying $\leq 6$ months, and the WBRT-30-SCLC to identify patients surviving $\geq 6$ months.

Keywords: Small-cell lung cancer (SCLC); elderly patients; cerebral metastases; remaining lifespan; diagnosisspecific tools

Submitted Feb 24, 2020. Accepted for publication Jun 30, 2020.

doi: $10.21037 /$ tlcr-20-327

View this article at: http://dx.doi.org/10.21037/tlcr-20-327 


\section{Introduction}

Of cancer patients with cerebral metastases, those with lung cancer represent the largest group with about $50 \%$ (1). Of this group, approximately $25 \%$ have small-cell lung cancer (SCLC); and of these patients, about $40 \%$ are elderly patients, generally defined as 65 years or older $(2,3)$. Due to demographic change, this group is constantly increasing and requires particular attention. It is generally agreed that elderly cancer patients have to be considered a separate group (4). Due to significant comorbidities and reduced functions of liver, kidneys and bone marrow, a considerably number of these patients may not be able to tolerate aggressive systemic and local therapies. Since the biological age may differ considerably from the numeric age, physicians need to look carefully at elderly patients as individual persons taking into account the biological age and potential personal resources (2-4). Thus, elderly patients may benefit from the comparatively novel strategy of treatment personalization. When designing a personalized treatment program, several factors need to be considered including the patient's remaining lifespan. If this is short, the treatment program should be at least burdensome and time-consuming as possible. When treating elderly patients with a longer remaining lifespan, long-term outcomes in terms of late toxicities and disease control become more important. This applies particularly to elderly patients assigned to radiotherapy for a palliative situation like cerebral metastasis from SCLC. The majority of these patients are treated with whole-brain irradiation (WBI), either alone or in combination with a local therapy such as radiosurgery, fractionated stereotactic radiotherapy or neurosurgical resection (5). Treatments with local therapies alone are generally limited to patients with very few lesions $(5,6)$. Compared to other primary tumors, patients with SCLC considerably more often receive WBI alone, because SCLC comparably often spreads to the brain and causes multiple lesions (5). This applies also to elderly patients who often have a reduced performance status. If an elderly patient is assigned to WBI alone, several dose-fractionation programs are available with overall treatment times usually ranging between one and four weeks (5). In order to select the most appropriate WBI-program, it is very important to be able to judge the patient's remaining life span. To support physicians during the process of treatment personalization for elderly patients with cerebral metastases from SCLC, a simple and specific tool was developed for estimation of the remaining lifespan of such patients. In addition, this new tool was compared to instruments that were created for patients with cerebral metastases from SCLC of any age and not specifically for elderly patients (7-9). We present the following article in accordance with the TRIPOD reporting checklist (available at http://dx.doi.org/10.21037/tlcr-20327).

\section{Methods}

In this retrospective cohort study, the data of 140 elderly patients ( $\geq 65$ years of age) irradiated for cerebral metastases from SCLC between January 1999 and December 2018 were evaluated with respect to survival. Patients were followed until death or for at least 6 months following WBI. Data were obtained from an existing anonymized database or from consecutive patients of two Northern German centers (anonymized data provided for survival analyses). Thus, data were blinded to the person who performed the survival analyses. All patients had received WBI alone for brain metastases from SCLC, either with 20 Gy in 5 fractions $(n=23), 30$ Gy in 10 fractions $(n=76)$ or total doses $>30$ Gy (35-40 Gy in 14-20 fractions, $n=41$ ). The present study was approved by the Ethic Committee of the University of Lübeck under the reference number AZ19-011A. The WBI-program plus six pre-treatment characteristics potentially associated with survival had been evaluated (Table 1). These pre-treatment characteristics included age at the beginning of WBI ( $\leq 72 v s . \geq 73$ years, median age: 72 years), gender (female $v s$. male), Karnofsky performance score (KPS) ( $<70 v s . \geq 70$, median score: $70)$, number of cerebral lesions (1-3vs. $\geq 4$ or more), extracerebral metastases prior to WBI (no vs. yes), and interval between diagnosis of SCLC and beginning of WBI ( $\leq 1 v s .>1$ month, median interval: 1 month). The data regarding these characteristics were complete for all patients.

For the univariate analyses of survival following WBI, we used the Kaplan-Meier-method. The differences of the Kaplan-Meier curves of each characteristic were obtained with the log-rank test.

When applying Bonferroni adjustment for seven tests, $\mathrm{P}$ values of $<0.007$ were considered significant representing an alpha level of $<0.05$. The characteristics, for which a significant association with survival $(\mathrm{P}<0.007)$ or a trend for such an association $(\mathrm{P}<0.06)$ was found, were additionally analyzed for independence using the Cox proportional hazard model. Those pre-treatment characteristics that were significant $(\mathrm{P}<0.05)$ in the analysis with the Cox 
Table 1 Characteristics evaluated for associations with survival

\begin{tabular}{lc}
\hline Characteristic & N patients (\%) \\
\hline Age & $79(56.4)$ \\
$\leq 72$ years & $61(43.6)$ \\
$\geq 73$ years & \\
Gender & $49(35.0)$ \\
Female & $91(65.0)$ \\
Male & \\
Karnofsky performance score & $61(43.6)$ \\
$<70$ & $79(56.4)$ \\
$\geq 70$ & \\
Number of cerebral lesions & $36(25.7)$ \\
1-3 & $104(74.3)$ \\
$\geq 4$ & \\
Extracerebral metastases & \\
No & \\
Yotal doses $>30$ Gy & \\
Interval between first diagnosis of SCLC & \\
and beginning of WBI & \\
\hline & \\
\hline & \\
\hline &
\end{tabular}

SCLC, small-cell lung cancer; WBI, whole-brain irradiation.

proportional hazard model were included in the prognostic tool. For each independent prognostic factor (pretreatment characteristic), the scoring points were calculated by dividing the 6 -month survival rate (in \%) by 10 . The scoring points of the characteristics were added, and the total scores for each patient were received.

In addition, the new tool was compared to existing diagnosis-specific instruments created for patients with cerebral metastases from SCLC of any age, namely the updated diagnosis-specific graded prognostic assessment classification (DS-GPA), the Rades-SCLC score and the WBRT-30-SCLC score (7-9). Comparisons were performed with respect to the positive predictive values (PPVs) to correctly identify patients who die within
6 months following WBI (comparison of the least favorable groups) and patients who survive for at least 6 months following WBI (comparison of the most favorable groups). The PPVs were calculated by dividing the number of true positives through (the number of true positives plus the number of false positives).

\section{Ethics approval}

All procedures performed were in accordance with ethical standards and the Helsinki declaration from 1964 as revised in 2013. The study was approved by an institutional review board (IRB), namely the Ethics Committee of the University of Lübeck, (reference number 18-254A). Due to its retrospective design, informed consent specifically for this study was not required by the IRB. For protection of to the patient's personal data, only anonymized data have been used for the analyses of this study

\section{Results}

Median follow up was 2 months (range, 0-22 months) in the entire series and 7 months (range, 6-13 months) in patients alive at their last follow up. In the entire cohort of 140 patients, 129 patients (92\%) died during the period of follow up, 114 patients (81\%) within 6 months following WBI. Survival rates at 3, 6, 9 and 12 months were $36 \%$, $19 \%, 10 \%$ and $8 \%$, respectively. On univariate analysis, the WBI-program $(\mathrm{P}=0.016)$, age $(\mathrm{P}=0.006)$, KPS $(\mathrm{P}<0.001)$, the number of cerebral lesions $(\mathrm{P}<0.001)$ and extracerebral metastases $(\mathrm{P}<0.001)$ showed significant associations with survival (Table 2). In addition, gender showed a trend $(\mathrm{P}=0.055)$.

In the multivariate analysis with the Cox proportional hazard model, KPS $(\mathrm{P}<0.001)$, the number of cerebral lesions $(\mathrm{P}=0.013)$ and extracerebral metastases $(\mathrm{P}=0.049)$ were significantly associated with survival (Table 3) and included in the prognostic tool. The corresponding scoring points for these three characteristics as obtained from the 6-month survival rates are shown in Table 4. When adding the scoring points for each patient, total scores were $2(n=37), 5(n=69), 8(n=20)$ or $11(n=14)$ points. The corresponding 6-month survival rates were $0 \%, 9 \%$, $50 \%$ and $78 \%$, respectively $(\mathrm{P}<0.001)$, and the median survival times 1, 2, 6 and 7 months, respectively $(\mathrm{P}<0.001)$. The survival rates up to 12 months following WBI are summarized in Table 5.

The PPV of the worst prognostic group (2 points) of the 
Table 2 Univariate analyses of survival of the seven evaluated characteristics

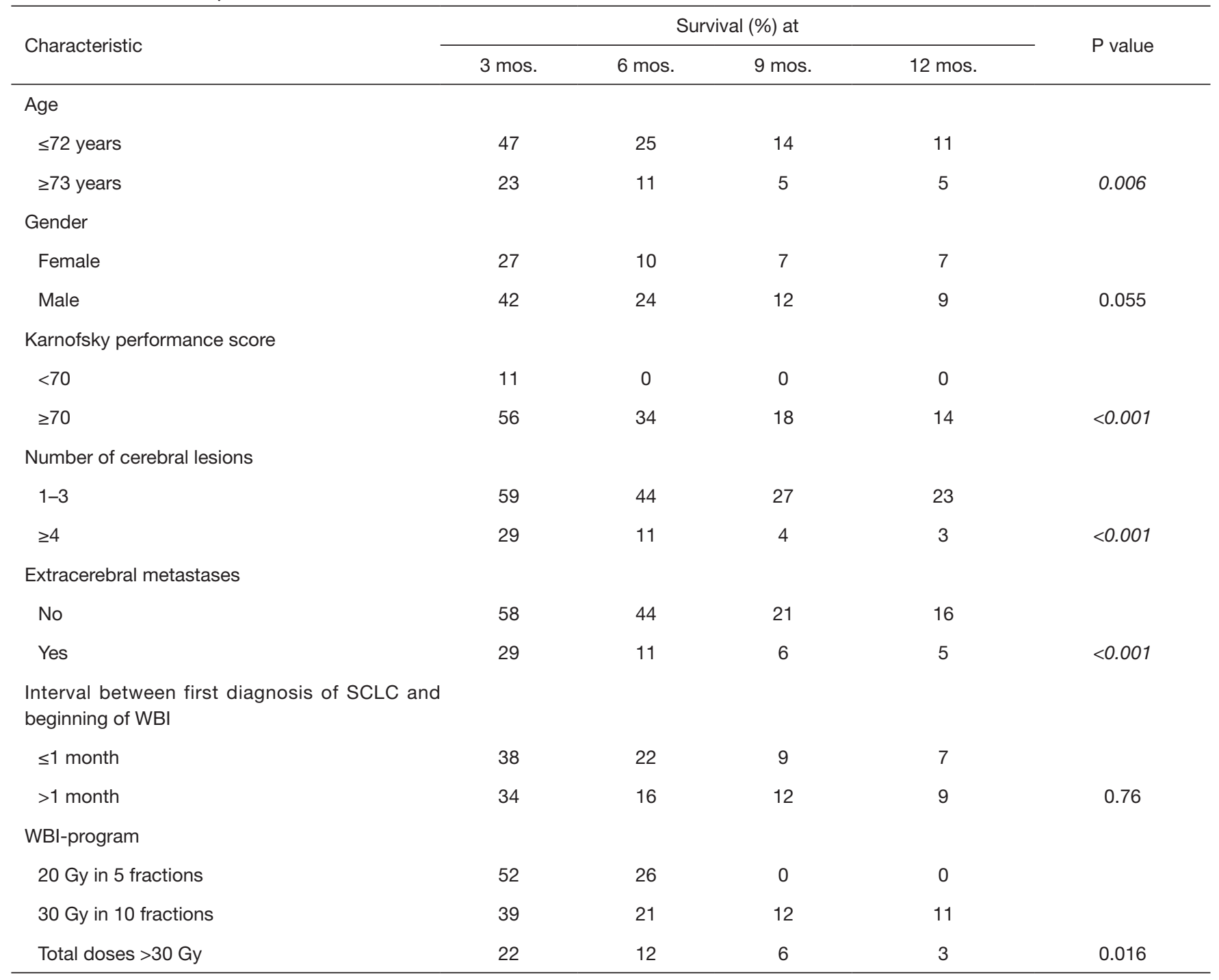

When applying Bonferroni adjustment, $\mathrm{P}$ values of $<0.0071$ were considered significant and are given in italic. SCLC, small-cell lung cancer; WBI, whole-brain irradiation.

Table 3 Results of the multivariate analysis of the characteristics showing at least a trend $(\mathrm{P}<0.06)$ on univariate analysis for an association with survival

\begin{tabular}{lccc}
\hline Characteristic & Hazard ratio & 95\% confidence interval & P value \\
\hline Age & 1.33 & $0.92-1.90$ & 0.13 \\
Gender & 1.34 & $0.91-1.94$ & 0.14 \\
Karnofsky performance score & 2.53 & $1.69-3.83$ & $<0.001$ \\
Number of cerebral lesions & 1.19 & $1.04-1.39$ & 0.013 \\
Extracerebral metastases & 1.55 & $1.00-2.48$ & 0.049 \\
WBI-program & 1.33 & $0.99-1.79$ & 0.06 \\
\hline
\end{tabular}

Significant $P$ values are given in italic. 
Table 4 Six-month survival rates of the independent prognostic factors and the corresponding scoring points

\begin{tabular}{lcc}
\hline Prognostic factor & 6-month survival rate & Scoring points \\
\hline Karnofsky performance score & 0 & 0 \\
$<70$ & 34 & 3 \\
$\geq 70$ & & 4 \\
Number of cerebral lesions & 44 & 1 \\
$1-3$ & 11 & 4 \\
$\geq 4$ & & 1 \\
Extracerebral metastases & 44 & 11 \\
No & & 4 \\
\hline
\end{tabular}

Table 5 Survival rates related to the total scores (2, 5,8 or 11 points) for individual patients

\begin{tabular}{lcccc}
\hline & \multicolumn{4}{c}{ Survival $(\%)$ at } \\
\cline { 2 - 5 } Prognostic group & 3 mos. & 6 mos. & 9 mos. & 12 mos. \\
\hline 2 points $(n=37)$ & 11 & 0 & 0 & 0 \\
5 points $(n=69)$ & 30 & 9 & 3 & 1 \\
8 points $(n=20)$ & 65 & 50 & 20 & 15 \\
11 points $(n=14)$ & 93 & 79 & 29 & 14 \\
\hline
\end{tabular}

Significant $P$ value is given in italic.

Table 6 Comparison of the new prognostic tool and existing tools with respect to the positive predictive values (PPVs) for correctly identifying patients dying within 6 months and surviving for at least 6 months following WBI. Existing tools included the DS-GPA classification (7), the Rades-SCLC score (8) and the WBRT-30-SCLC score (9)

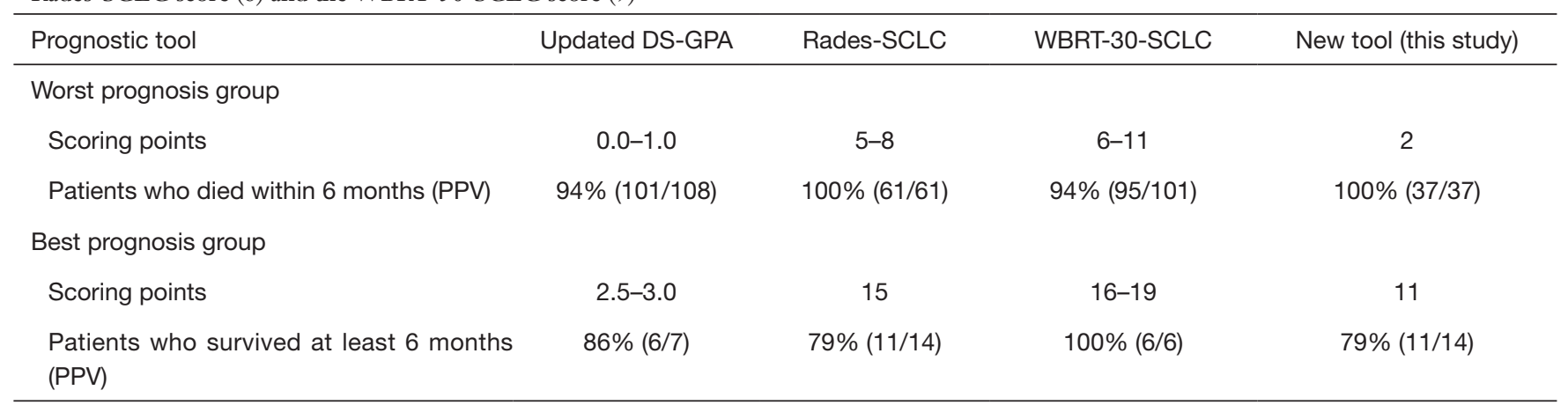

DS-GPA, diagnosis-specific graded prognostic assessment; SCLC, small-cell lung cancer; WBRT, whole-brain radiotherapy; SCLC, smallcell lung cancer; WBI, whole-brain irradiation.

present score to identify patients dying within 6 months following WBI was $100 \%$. The same PPV (100\%) was achieved with the Rades-SCLC score, and the PPVs of the updated DS-GPA classification and the WBRT-30-SCLC score were $94 \%$ and $94 \%$, respectively. The PPV of the most favorable prognostic group (11 points) of the present score to identify patients surviving 6 months or longer following WBI was 79\%. The corresponding PPVs of most 
favorable groups of the Rades-SCLC score, the updated DS-GPA classification and the WBRT-30-SCLC score were $79 \%, 86 \%$ and $100 \%$, respectively. The comparisons of the four prognostic tools are summarized in Table 6.

\section{Discussion}

Elderly patients with cerebral metastases often have poor survival prognoses that need considerable improvement (4). This goal may be achieved with the introduction of novel targeted systemic agents including tyrosine kinase inhibitors and checkpoint inhibitors. However, these agents are mainly used for patients with non-small cell lung cancer (NSCLC) rather than for patients with SCLC (10-14). For patients with cerebral metastases from SCLC, WBI alone still is the most frequently used type of treatment, particularly for elderly patients who may not be able to tolerate novel systemic agents that can cause considerable side effects including severe inflammatory reactions (5). Another option to improve the outcomes of elderly patients with cerebral metastases from SCLC is the comparably novel strategy of treatment personalization. Tailoring an individual program to a patients helps avoiding under- and over-treatment. When designing a personalized treatment program, several factors should be taken into account including the patient's personal preferences regarding the intensity of the treatment, as well as the patient's general condition, comorbidities and social environment. In addition, the patient's remaining lifespan needs to be regarded (4). To be aware of an individual patient's prognosis, survival scores have been created for different settings including palliative situations like the presence of cerebral metastases. It is generally agreed that specific tools for the different primary tumor types would be important to adequately take into account the specific biological behavior of each tumor type. Moreover, elderly patients represent a specific group of cancer patients and may require separate scoring tools. In the present study, a specific tool has been created for elderly patients with cerebral metastases from SCLC.

Based on three independent prognostic factors, namely KPS, number of cerebral lesions and extracerebral metastases, four different scores (2, 5, 8 and 11 points) were obtained. Patients with 0 points had a median survival time of only 1 month, and only $11 \%$ of the patients survived for 3 months. Considering their extraordinarily poor prognoses, these patients may be considered for best supportive care including corticosteroids without WBI. A randomized trial of 538 patients with cerebral metastases from NSCLC and very poor survival did not demonstrate a significant difference with respect to overall survival, overall quality of life and the use of dexamethasone between patients receiving short-course WBI with 20 Gy in 5 fractions plus best supportive care and patients receiving best supportive care alone (15). The mean quality-adjusted lifeyears were 46.4 days with WBI and 41.7 days without WBI, respectively. Against the background of our present study, it is remarkable that more than half of the patients included in that randomized trial were elderly patients (median age: 66 years) (15).

In the present study, the median survival time of the patients who achieved 5 points was 2 months, and $30 \%$ of the patients survived for 3 months or longer. These patients should be considered for short-course WBI with 20 Gy in 5 fractions, since in a previous study of 442 patients of any age and with different primary tumor types 20 Gy in 5 fractions over 1 week was not inferior to $30 \mathrm{~Gy}$ in 10 fractions over 2 weeks with respect to cerebral control $(\mathrm{P}=0.07)$ and survival $(\mathrm{P}=0.29)(16)$. Selected patients of the 5 -points group may also be candidates for best supportive care plus corticosteroids without WBI (15) Patients of the 8-points group had more favorable prognoses with a median survival time of 6 months and a 6 -month survival rate of $50 \%$. These patients appear good candidates for longer-course WBI with $30 \mathrm{~Gy}$ in 10 fractions, which is the most common WBI-program worldwide and may be considered the "standard" program, or even for longercourse programs with total doses $>30$ Gy and doses per fraction of $<3$ Gy. Lower dose per fractions were reported to result in less pronounced WBI-induced decline in neuro-cognitive function (17). Neuro-cognitive decline is considered a late toxicity, and the risk of developing this toxicity increases with lifetime. Moreover, patients with most favorable prognoses, i.e., patients of the 11-points group in the current study, can benefit from 40 Gy in 20 fractions over 4 weeks. In a previous study of 174 patients with cerebral metastases and favorable survival prognoses, $40 \mathrm{~Gy}$ in 20 fractions when compared to $30 \mathrm{~Gy}$ in 10 fractions resulted in better 1-year cerebral control (44\% vs. $28 \%, \mathrm{P}=0.047$ in the multivariate analysis) and survival (61\% vs. $50 \%, \mathrm{P}=0.008)(18)$. Moreover, in order to reduce the risk of neuro-cognitive decline, patients of the 8-points and the 11-points group should be considered for hippocampal sparing. According to previous studies, this modern technique can significantly decrease the rate of neurocognitive deficits following WBI without significantly increasing the risk of new cerebral metastases $(19,20)$.

In the second part of the present study, the new scoring 
tool was compared to three existing diagnosis-specific instruments designed from patients with cerebral metastases from SCLC of any age (7-9). The four tools were compared regarding the PPVs to correctly identify patients dying within 6 months and patients surviving for at least 6 months. The highest PPV with respect to the identification of patients dying within 6 months were found for the new tool and our previous tool not particularly developed for elderly patients (Rades-SCLC). Both tools achieved the highest possible PPV of $100 \%$ correct prediction. When aiming to predict the probability of surviving for at least 6 months following WBI, the WBRT30-SCLC was the most accurate tool and also achieved a PPV of $100 \%$, whereas the other three tools ranged between $79 \%$ and $86 \%$. Thus, depending on what one wishes to estimate, the new tool, the Rades-SCLC or the WBRT-30-SCLC appear the most appropriate tools. However, when using these prognostic instruments, on should be aware that they were created from retrospective data, which always bear the risk of hidden selection biases. Moreover, validation of the tools, ideally in a prospective cohort of patients, is warranted.

\section{Conclusions}

A new and simple tool was developed particularly for elderly patients receiving WBI for cerebral metastases from SCLC. This new tool achieved the highest possible PPV for correct prediction of death within 6 months after WBI, which was also achieved by the previous Rades-SCLC score created in patients of any age. The most precise instrument for identification of patients surviving for at least 6 months was the WBRT-30-SCLC score.

\section{Acknowledgments}

Funding: None.

\section{Footnote}

Reporting Checklist: The authors have completed the TRIPOD reporting checklist. Available at http://dx.doi. org/10.21037/tlcr-20-327

Data Sharing Statement: Available at http://dx.doi. org/10.21037/tlcr-20-327

Conflicts of Interest: All authors have completed the ICMJE uniform disclosure form (available at http://dx.doi. org/10.21037/tlcr-20-327). The authors have no conflicts of interest to declare.

Ethical Statement: The authors are accountable for all aspects of the work in ensuring that questions related to the accuracy or integrity of any part of the work are appropriately investigated and resolved. All procedures performed were in accordance with ethical standards and the Helsinki declaration from 1964 as revised in 2013. The study was approved by an institutional review board (IRB), namely the Ethics Committee of the University of Lübeck (reference number 18-254A). Due to its retrospective design, informed consent specifically for this study was not required by the IRB. For protection of to the patient's personal data, only anonymized data have been used for the analyses of this study.

Open Access Statement: This is an Open Access article distributed in accordance with the Creative Commons Attribution-NonCommercial-NoDerivs 4.0 International License (CC BY-NC-ND 4.0), which permits the noncommercial replication and distribution of the article with the strict proviso that no changes or edits are made and the original work is properly cited (including links to both the formal publication through the relevant DOI and the license). See: https://creativecommons.org/licenses/by-nc-nd/4.0/.

\section{References}

1. Siegel RL, Miller KD, Jemal A. Cancer statistics, 2019. CA Cancer J Clin 2019;69:7-34.

2. Shenoy P, Harugeri A. Elderly patients' participation in clinical trials. Perspect Clin Res 2015;6:184-9.

3. Homepage of the World Health Organization (WHO) Available online: http://www.who.int/healthinfo/survey/ ageingdefnolder/en

4. Evers JN, Schild SE, Segedin B, et al. A new score predicting survival prognosis after whole-brain radiotherapy alone for brain metastases in elderly patients. Anticancer Res 2014;34:2455-8.

5. Tsao MN, Rades D, Wirth A, et al. Radiotherapeutic and surgical management for newly diagnosed brain metastasis(es): An American Society for Radiation Oncology evidence-based guideline. Pract Radiat Oncol 2012;2:210-25.

6. Rades D, Huttenlocher S, Dziggel L, et al. A new tool predicting survival after radiosurgery alone for one 
or two cerebral metastases from lung cancer. Lung 2015;193:299-302.

7. Sperduto PW, Kased N, Roberge D, et al. Summary report on the graded prognostic assessment: an accurate and facile diagnosis-specific tool to estimate survival for patients with brain metastases. J Clin Oncol 2012;30:419-25.

8. Rades D, Dziggel L, Segedin B, et al. The first survival score for patients with brain metastases from small cell lung cancer (SCLC). Clin Neurol Neurosurg 2013;115:2029-32.

9. Rades D, Hansen HC, Janssen S, et al. Comparison of diagnosis-specific survival scores for patients with smallcell lung cancer irradiated for brain metastases. Cancers (Basel) 2019;11:233.

10. Spagnuolo A, Muto M, Monaco F, et al. The optional approach of oncogene-addicted non-small cell lung cancer with brain metastases in the new generation targeted therapies era. Transl Lung Cancer Res 2019;8:1134-51.

11. Nishino M, Soejima K, Mitsudomi T. Brain metastases in oncogene-driven non-small cell lung cancer. Transl Lung Cancer Res 2019;8:S298-S307.

12. Dong K, Liang W, Zhao S, et al. EGFR-TKI plus brain radiotherapy versus EGFR-TKI alone in the management of EGFR-mutated NSCLC patients with brain metastases. Transl Lung Cancer Res 2019;8:268-79.

13. Zhuang H, Shi S, Chang JY. Treatment modes for EGFR mutations in patients with brain metastases from nonsmall cell lung cancer: controversy, causes, and solutions. Transl Lung Cancer Res 2019;8:524-31.

14. Kim R, Keam B, Kim S, et al. Differences in tumor microenvironments between primary lung tumors and brain metastases in lung cancer patients: therapeutic

Cite this article as: Rades D, Nguyen T, Janssen S, Schild SE. Development of a multivariable prediction model to estimate the remaining lifespan of elderly patients with cerebral metastases from small-cell lung cancer. Transl Lung Cancer Res 2020;9(4):1433-1440. doi: 10.21037/tlcr-20-327 implications for immune checkpoint inhibitors. BMC Cancer 2019;19:19.

15. Mulvenna P, Nankivell M, Barton R, et al. Dexamethasone and supportive care with or without whole brain radiotherapy in treating patients with non-small cell lung cancer with brain metastases unsuitable for resection or stereotactic radiotherapy (QUARTZ): results from a phase 3, non-inferiority, randomised trial. Lancet 2016;388:2004-14.

16. Rades D, Kieckebusch S, Lohynska R, et al. Reduction of overall treatment time in patients irradiated for more than three brain metastases. Int J Radiat Oncol Biol Phys 2007;69:1509-13.

17. DeAngelis LM, Delattre JY, Posner JB. Radiation-induced dementia in patients cured of brain metastases. Neurology 1989;39:789-96.

18. Rades D, Panzner A, Dziggel L, et al. Dose-escalation of whole-brain radiotherapy for brain metastasis in patients with a favorable survival prognosis. Cancer 2012;118:3852-9.

19. Gondi V, Pugh SL, Tome WA, et al. Preservation of memory with conformal avoidance of the hippocampal neural stem-cell compartment during whole-brain radiotherapy for brain metastases (RTOG 0933): a phase II multi-institutional trial. J Clin Oncol 2014;32:3810-6.

20. Kundapur V, Ellchuk T, Ahmed S, et al. Risk of hippocampal metastases in small cell lung cancer patients at presentation and after cranial irradiation: a safety profile study for hippocampal sparing during prophylactic or therapeutic cranial irradiation. Int J Radiat Oncol Biol Phys 2015;91:781-6. 